\title{
Treatment of early caries lesions using biomimetic self-assembling peptides - a clinical safety trial
}

\author{
P. A. Brunton, ${ }^{1}$ R. P. W. Davies, ${ }^{2}$ J. L. Burke, ${ }^{3}$ A. Smith,${ }^{4}$ A. Aggeli, ${ }_{1}$ \\ S. J. Brookes ${ }^{5}$ and J. Kirkham ${ }^{* 5}$
}

IN BRIEF

- Describes a rationally-designed peptidebased biomaterial capable of infiltrating early caries lesions where it is thought to form scaffold-like structures via a process of self-directed assembly.

- Suggests that peptide scaffolds promote enamel regeneration in situ within the lesion, potentially providing a new therapeutic option that is minimally invasive and highly acceptable to patients.

\section{VERIFIABLE CPD PAPER}

\begin{abstract}
Objective We previously reported that a rationally designed biomimetic self-assembling peptide, $\mathrm{P}_{11}-4$, nucleated hydroxyapatite de novo and was apparently capable of in situ enamel regeneration following infiltration into caries-like lesions. Our present aim was to determine the safety and potential clinical efficacy of a single application of $\mathrm{P}_{11}-4$ on early enamel lesions. Materials and methods Fifteen healthy adults with Class V 'white spot' lesions received a single application of $\mathrm{P}_{11}-4$. Adverse events and lesion appearances were recorded over 180 days. Results Patients treated with $\mathrm{P}_{11}-4$ experienced a total of 11 adverse events during the study, of which two were possibly related to the protocol. Efficacy evaluation suggested that treatment with $\mathrm{P}_{11}-4$ significantly decreased lesion size $(p=0.02)$ after 30 days and shifted the apparent progression of the lesions from 'arrested/progressing' to 'remineralising' $(p<0.001)$. A highly significant improvement in the global impression of change was recorded at day 30 compared with baseline $(p<0.001)$. Conclusions The results suggest that treatment of early caries lesions with $\mathrm{P}_{11}-4$ is safe, and that a single application is associated with significant enamel regeneration, presumably by promoting mineral deposition within the subsurface tissue.
\end{abstract}

\section{INTRODUCTION}

We previously described the effect of application of an anionic self-assembling peptide, $\mathrm{P}_{11}-4$, on the remineralisation and demineralisation behaviour of carieslike lesions under simulated intra-oral conditions. ${ }^{1}$ Peptide treatment significantly increased net mineral gain due to a combined effect of increased mineral gain and inhibition of mineral loss. In addition, $\mathrm{P}_{11}-4$ in its assembled form was shown to induce hydroxyapatite nucleation de novo. Based upon these data, we predicted that self-assembling peptides would be useful in the modulation of mineral behaviour during in situ dental tissue engineering.

$\mathrm{P}_{11}-4$ is a rationally-designed self-assembling peptide. ${ }^{2,3}$ Self-assembling peptides

${ }^{1}$ Restorative Dentistry, ${ }^{3}$ Oral Surgery, ${ }^{5}$ Biomineralisation Research Group, Leeds Dental Institute, University of Leeds, Clarendon Way, Leeds, LS2 9LU; ${ }^{2}$ Centre for Molecular Nanoscience, School of Chemistry, University of Leeds, Leeds, LS2 9JT; ${ }^{4}$ York Health Economics Consortium, Market Square, University of York, Heslington, York, Y010 5NH

${ }^{*}$ Correspondence to: Professor Jennifer Kirkham Email: J.Kirkham@Leeds.ac.uk; Tel: 44 (0) 1133436156

\section{Online article number E6}

Refereed Paper - accepted 2 July 2013

DOI: 10.1038/sj.bdj.2013.741

${ }^{\circledR}$ British Dental Journal 2013; 215: E6

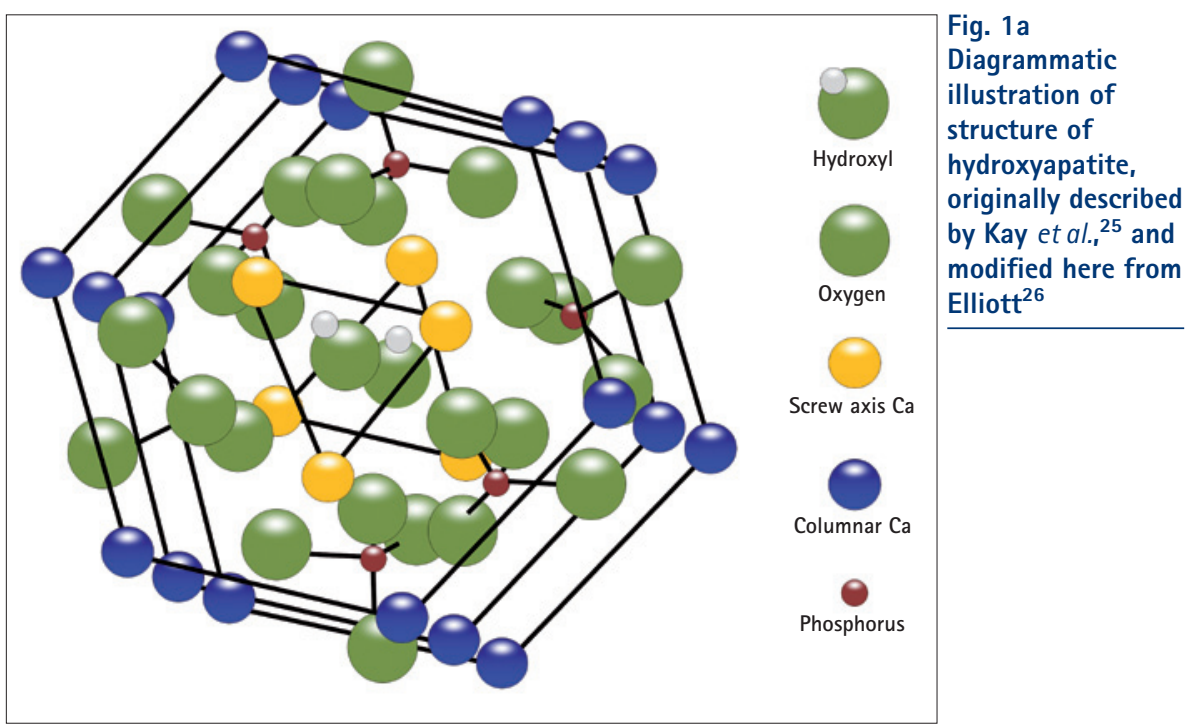

undergo well-characterised hierarchial self-assembly into three-dimensional fibrillar scaffolds in response to specific environmental triggers, ${ }^{4-6}$ offering a new generation of well-defined biopolymers with a range of potential applications. ${ }^{7}$ At peptide concentrations used here, $\mathrm{P}_{11}-4$ switches from a low viscosity isotropic liquid to an elastomeric nematic gel at $\mathrm{pH}<7.4$ and in the presence of cations, conditions presumed to be found within a caries lesion. ${ }^{8}$ In a number of in vitro and in vivo experiments, the assembled $\mathrm{P}_{11}-4$ fibres were shown to be highly biocompatible with low immunogenicity. ${ }^{9,10}$

Assembled $\mathrm{P}_{11}-4$ forms scaffold-like structures with negative charge domains, mirroring biological macromolecules in mineralised tissue extracellular matrices (ECM). ${ }^{11}$ Proteins of the developing enamel ECM - themselves known to form self-assembling supramolecular structures $^{12}$ - have long been thought to control initial mineral deposition ('nucleation') and subsequent crystal growth, ultimately determining the physico-mechanical 
properties of the mature tissue. ${ }^{13}$ Mutations in enamel extracellular matrix proteins result in enamel defects in amelogenesis imperfecta, underlining the importance of protein-mineral interactions during enamel biomineralisation. ${ }^{14,15}$ Nucleation of mineral de novo is the first step in both the biomineralisation process and when designing biomaterials for mineralised tissue regeneration. Dental enamel mineral is comprised of a substituted hydroxyapatite, $\mathrm{Ca}_{10}\left(\mathrm{PO}_{4}\right)_{6}(\mathrm{OH})_{2}$ (Fig. 1a), with carbonate, fluoride and magnesium being the most common heteroionic substitutents. ${ }^{16}$ Nucleators act to bring together constituent mineral ions from the surrounding disorganised ionic mileu within the tissue fluids into a highly ordered crystal lattice structure by stabilising critical nucleii to permit crystal growth. In order to control the deposition of hydroxyapatite crystals during biomineralisation, critical ionic nuclei need to be formed by collision of relevant ions. Such nuclei need to be stable before subsequent crystal growth (Fig. 1b). Proteins of the extracellular matrices of mineralised tissues, which can bind constituent ions and stabilise critical nuclei, thus reducing the activation energy required ('heterogeneous nucleation') are important in the biological control of biomineralisation.

In a biomimetic strategy based on recapitulating normal enamel histogenesis, we proposed that following $\mathrm{P}_{11}-4$ self-assembly, the anionic groups of the $\mathrm{P}_{11}-4$ side chains would attract $\mathrm{Ca}^{++}$ions, inducing de novo precipitation of hydroxyapatite as indicated in our earlier study. ${ }^{1}$

Enamel caries is a progressive subsurface demineralisation ultimately resulting in mechanical failure and cavitation. ${ }^{17}$ Invasive surgical treatment for enamel restoration has changed little in the past decades, despite advances in dental materials themselves. The earliest clinical sign of enamel caries is the appearance of a 'white spot' lesion on the tooth surface. ${ }^{18}$ At this stage, clinicians frequently elect to monitor lesion appearance, perhaps after the use of topical fluorides, to determine whether or not the lesion will progress, in which case a restoration would then be placed. Non-surgical intervention promoting defect biomineralisation or regeneration at the white spot lesion stage would remove the need to 'wait and see' and
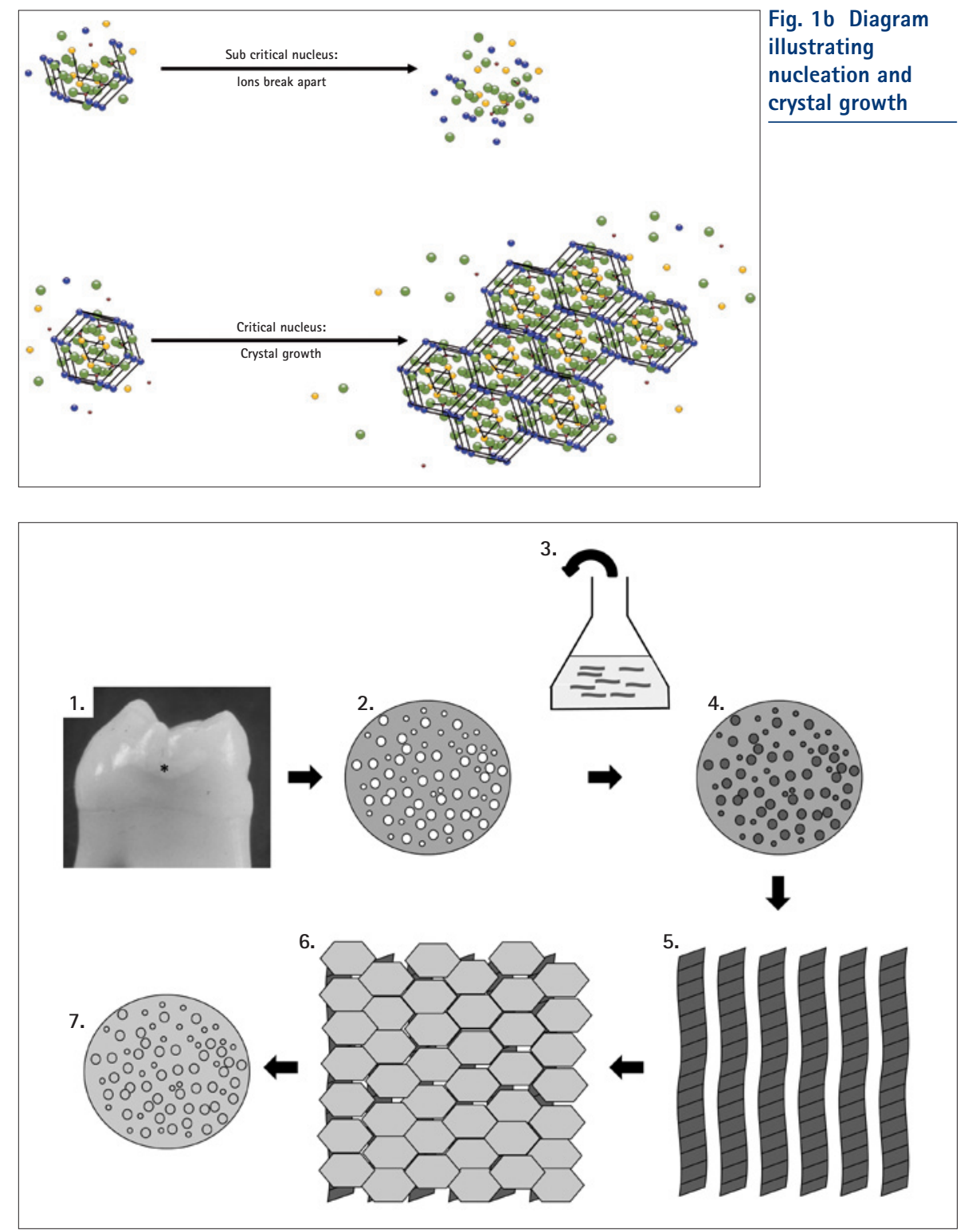

Fig. 2 Schematic showing underlying hypothesis for treatment of early caries using $P_{11}-4$ self assembling peptides. (1) Early caries appears as a 'white spot' on the enamel surface $(*)$, this is due to the underlying porosity in the tissue (2). Aqueous $P_{11}-4$ in its monomeric form applied to the lesion surface (3) will penetrate in to the pores due to its low viscosity (4). Self-assembly is triggered by the conditions ( $\mathrm{pH}<7.4$, presence of salts) within the lesion forming fibres (5) which can nucleate hydroxyapatite mineral (6). This restores both the enamel mineral and the natural appearance (7) of the original lesion

avoid the ultimate excavation of the tooth to place a restoration.

We hypothesised that infiltration of early ('white spot') caries lesions using low viscosity monomeric $\mathrm{P}_{11}-4$ would result in triggered self-assembly within the lesion, generating a subsurface bioactive scaffold capable of recapitulating normal histogenesis by inducing mineral deposition in situ (Fig. 2).

The specific aim of the present study was to conduct a first-in-man clinical safety study applying a single treatment of $\mathrm{P}_{11}-4$ to Class $\mathrm{V}$ enamel lesions in healthy human volunteers.

\section{MATERIALS AND METHODS}

\section{Preparation of $\mathrm{P}_{11}-4$}

HPLC purified peptide $\mathrm{P}_{11}-4\left(\mathrm{CH}_{3} \mathrm{CO}-\right.$ QQRFEWEFEQQ- $\mathrm{CONH}_{2}$ ) was acquired from PolyPeptide Group (France). Mass spectrometry found an experimental molecular weight of 1,595.8 (expected tent was determined by amino acid analysis and UV absorbance and found to be $89.2 \pm 2.5 \%$ (expected maximum peptide content $=90.5 \%$, calculated taking into account only bound counterions, not bound water). molecular weight $=1,595.7)$. Peptide con- 
Table 1 Tooth type, lesion position and lesion activity at baseline for teeth treated with $P_{11}-4$

\begin{tabular}{l|l|l} 
Patient-number & Tooth number and lesion position* & Lesion activity at baseline as judged by VAS*
\end{tabular}

\begin{tabular}{|c|c|c|}
\hline 1-001 & $36 b$ & -0.5 \\
\hline 1-002 & $46 b$ & 20.5 \\
\hline 1-003 & $46 b$ & 11 \\
\hline $1-004$ & $46 b$ & -11 \\
\hline $1-005$ & $46 b$ & -5.5 \\
\hline 1-006 & $46 b$ & -3.5 \\
\hline 1-007 & $46 b$ & 6 \\
\hline 1-008 & $46 b$ & -2.5 \\
\hline 1-009 & $16 b$ & 1.5 \\
\hline $1-010$ & $16 \mid$ & 15 \\
\hline 1-011 & $44 b$ & 7 \\
\hline 1-012 & $35 b$ & 3.5 \\
\hline 1-013 & $43 b$ & 9.5 \\
\hline 1-014 & $14 b$ & 9.5 \\
\hline $1-015$ & $24 b$ & -16.5 \\
\hline
\end{tabular}

$\mathrm{P}_{11}-4$ was dissolved in water $(18.2 \mathrm{M} \Omega-\mathrm{cm})$ at a concentration of $6.3 \mathrm{mM}$. A solution of $1 \%$ ammonia was added drop wise until a $\mathrm{pH}$ of $8.6 \pm 0.1$ was achieved. The sample was then vortexed and sonicated to produce a clear colourless monomeric fluid. Aliquots (0.05 ml) of monomeric $\mathrm{P}_{11}-4$ were pipetted into individual vials, flash frozen with liquid nitrogen and lyophilised for 24 hours. The individual samples were then sealed with a crimp.

\section{Selection of volunteers}

Ethical approval was obtained through the UK National Research Ethics System (NRES; project number 10/H1207/75). Subjects were recruited from staff and patients attending Leeds Dental Institute (LDI). The study was conducted according to ISO 14155:2003 in the University of Leeds Translational and Clinical Research Unit at LDI. Fifteen subjects with 19 lesions were recruited according to the following criteria: included in the study were:

- Subjects with $\geq 1$ visible and accessible early caries lesion present, which did not require operative intervention

- Subjects aged between 18 and 65 years who were willing and able to observe good oral hygiene and attend for the study visits

- Subjects with more than 20 teeth present and a Basic Periodontal Examination (BPE) score of less than three

- Subjects who were willing and able to understand all study-related procedures.

Excluded from the study were:

- Subjects with $>1$ caries lesion present on the selected tooth

- Subjects who had a significant medical history, including pregnancy and breast feeding or if they smoked more than five cigarettes per day

- Subjects who had evidence of reduced salivary flow or significant tooth wear

- Subjects who were participating in concurrent clinical trials or who had recently participated in other clinical trials of an investigational drug or device.

Mean subject age for the study group was $34.4 \pm 12.7$ years. The group comprised of seven females and eight males. Tooth type, lesion position and lesion activity are shown in Table 1.

\section{Baseline assessment (Day 1)}

The following baseline assessments were performed:

- A general oral and physical examination including a dental, medical and social history and vital signs

- A series of digital colour photographs of the test lesion taken under standard conditions of light and magnification

- A detailed relevant medical (including dental) history covering the previous three years and any medication they were taking at the time of the study.

At each recall the patients were questioned to determine whether or not they might have experienced any adverse events since their last visit and to identify any new medication(s) that they had taken since joining the trial.

\section{Clinical procedures}

The test lesion was cleaned with a prophylaxis paste (Kemdent Flour of Pumice, ADP Ltd, Swindon, UK) and isolated. The lesion was then treated with etch solution (Super Etch, SDI Ltd, Bayswater, Victoria, Australia) for 30 seconds to open up the pores to the subsurface lesion and subsequently washed and dried. Lyophilised $\mathrm{P}_{11}-4$ was rehydrated with $0.05 \mathrm{ml}$ of sterile water and a single drop of the resulting solution immediately applied directly to the lesion surface. Moisture control was ensured until the $\mathrm{P}_{11}-4$ solution was no longer visible (approximately two minutes).

Subjects were advised not to brush the quadrant containing the treated tooth until Day 4 (D4) review visit. Instead, subjects were asked to rinse that area of the mouth with a chlorhexidine mouthwash (Corsodyl, GSK, Herrenberg, Germany). At D4, a soft toothbrush and toothpaste were provided and subjects asked to use these until Day 8 (D8) review visit. At this point they reverted to their normal oral hygiene procedures.

\section{Review assessments (D4, D8, D30 and D180)}

Subjects were reviewed at 4, 8, 30 and 180 days after treatment. At each visit the following assessments were conducted:

- Visual inspection of the treated site was carried out and any potential adverse events recorded

- Vital signs were recorded 
- A series of colour photographs of the test lesion were taken under standard conditions of light and magnification

- At D30 and D180, a Global Impression of Change questionnaire was completed by both the subjects and dental professionals.

\section{Assessment of lesion appearance and progression}

Colour photographs of all lesions from all subjects were used to assess lesion size, appearance and progression. Blind assessment was carried out independently by two experienced clinicians using a global impression of change questionnaire and a visual analogue scale (VAS).

Interim assessment was carried out by comparing lesion size and appearance at D4, D8 and D30 to D1 (baseline). Final assessment was carried out by comparing D180 with D30 and correlating the data to the interim assessment.

Colour photographs for all assessments were selected by a member of staff who was not involved in the study and were each allocated a unique identifier including the day of the visit in order that comparisons with baseline might be made. As such, the assessors would not have been blind to the identity of the lesions at baseline. To address this, D1 and D30 photographs were 'switched' according to a randomisation table such that the assessors did not know whether they were making comparisons against 'true' D1 baseline. Neither assessor was aware which baseline data had been 'switched. Where 'switching' had occurred, a real improvement with time after treatment would appear as an apparent deterioration.

Unblinding was performed after completion of all assessments and database lock.

\section{Image analysis}

For each lesion, digital images were selected from photographs taken at baseline (D1), D30 and D180. Lesion contour was marked by a blinded operator and the total number of pixels within contour boundaries determined using image analysis (Soft Imaging System, Münster, Germany). Only those images where tooth orientation was consistent across the three visits were used (validated using tooth landmarks and a series of interconnecting lines) and hence a total of 12 (D1 \& D30) and 11 (D180) lesions were analysed.

\begin{tabular}{|c|c|c|}
\hline Patient number & Adverse event & Treatment causality \\
\hline $1-002$ & Chest infection & Not related \\
\hline $1-003$ & Dental hypersensitivity & Possibly related \\
\hline $1-003$ & Sprained joint between spine and pelvis & Not related \\
\hline $1-004$ & Stomach upset & Not related \\
\hline $1-005$ & Sensitivity with corsodyl mouthrinse & Possibly related \\
\hline $1-006$ & Diarrhoea and vomiting & Not related \\
\hline $1-007$ & Superficial staining & Not related \\
\hline $1-012$ & Chest infection & Not related \\
\hline $1-013$ & Dental treatment & Not related \\
\hline $1-015$ & Operation on Dupuytren's Contraction & Not related \\
\hline $1-015$ & Routine dental treatment & Not related \\
\hline
\end{tabular}

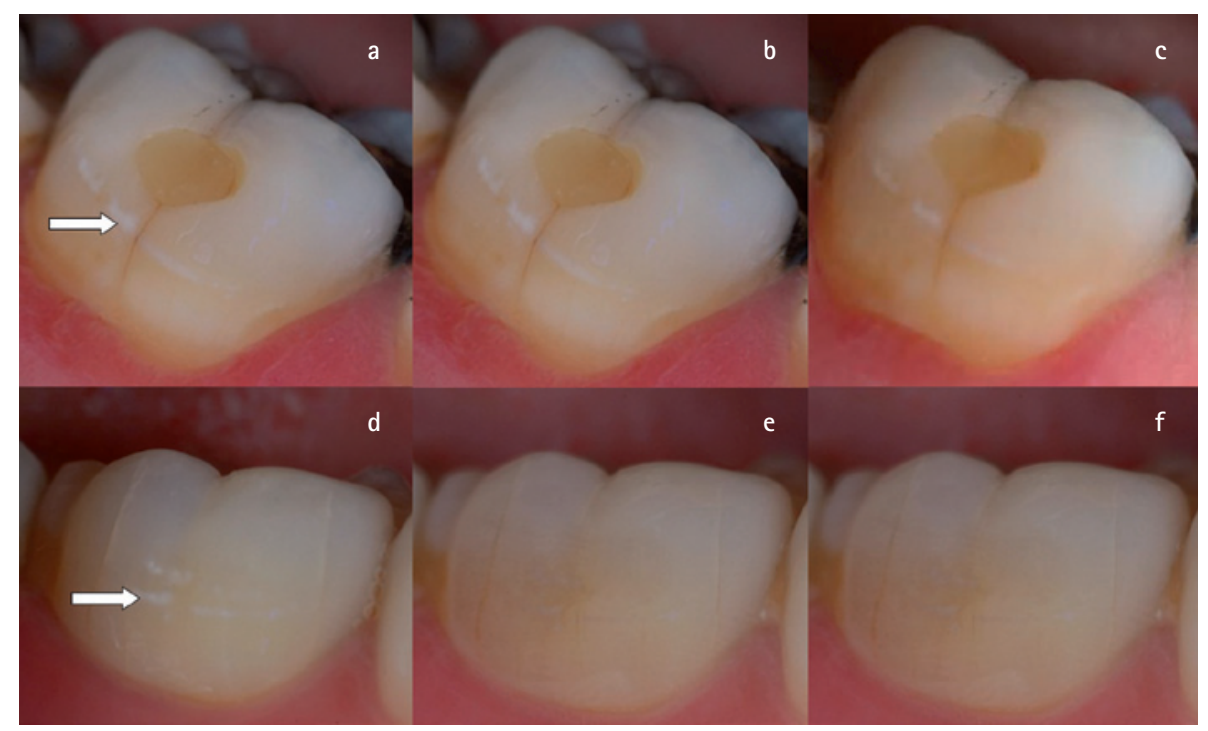

Fig. 3 Examples of clinical appearance of two Class V caries lesions (arrows) in different subjects included in the study. Images selected to show the range of response to a single application of $\mathrm{P}_{11}-4$ with time. ( $\left.a, d\right)$ Lesion appearance at baseline (DO); (b, e) and (c, $f$ ) appearance of same lesion at D30 and D180 respectively

\section{Statistical analysis}

Data was analysed by an independent statistician at the University of York.

Independent sample t-tests were used to determine any statistically significant differences between the two assessors' ratings for colour, size, progression and global impression of change.

A repeated measures t-test was used to assess statistically significant changes over time in the colour, size, progression assessments and the global impression of change. Fisher's method was used to combine p-values from the repeated measures t-tests for the two assessors, producing chi-squared test statistics that can be evaluated for statistical significance against $2 \mathrm{k}$ degrees of freedom for $\mathrm{k}$ number of tests.
The alpha value was adjusted to compensate for positively associated tests (that is, lack of independence) using $\mathrm{a}(\mathrm{k}+1) /(2 \mathrm{k})$, providing an alpha value of 0.0375 .

\section{RESULTS}

All subjects completed all stages of the study with the exception of one individual who was lost to follow-up at D180 due to their relocation outside of the area. There were 11 recorded adverse events (See Table 2) of which two were judged by the investigator as probably related to the protocol used in the trial. One was a transient dental hypersensitivity; the other a sensitivity to the Corsodyl mouthwash provided within the study. All other adverse events were judged to 

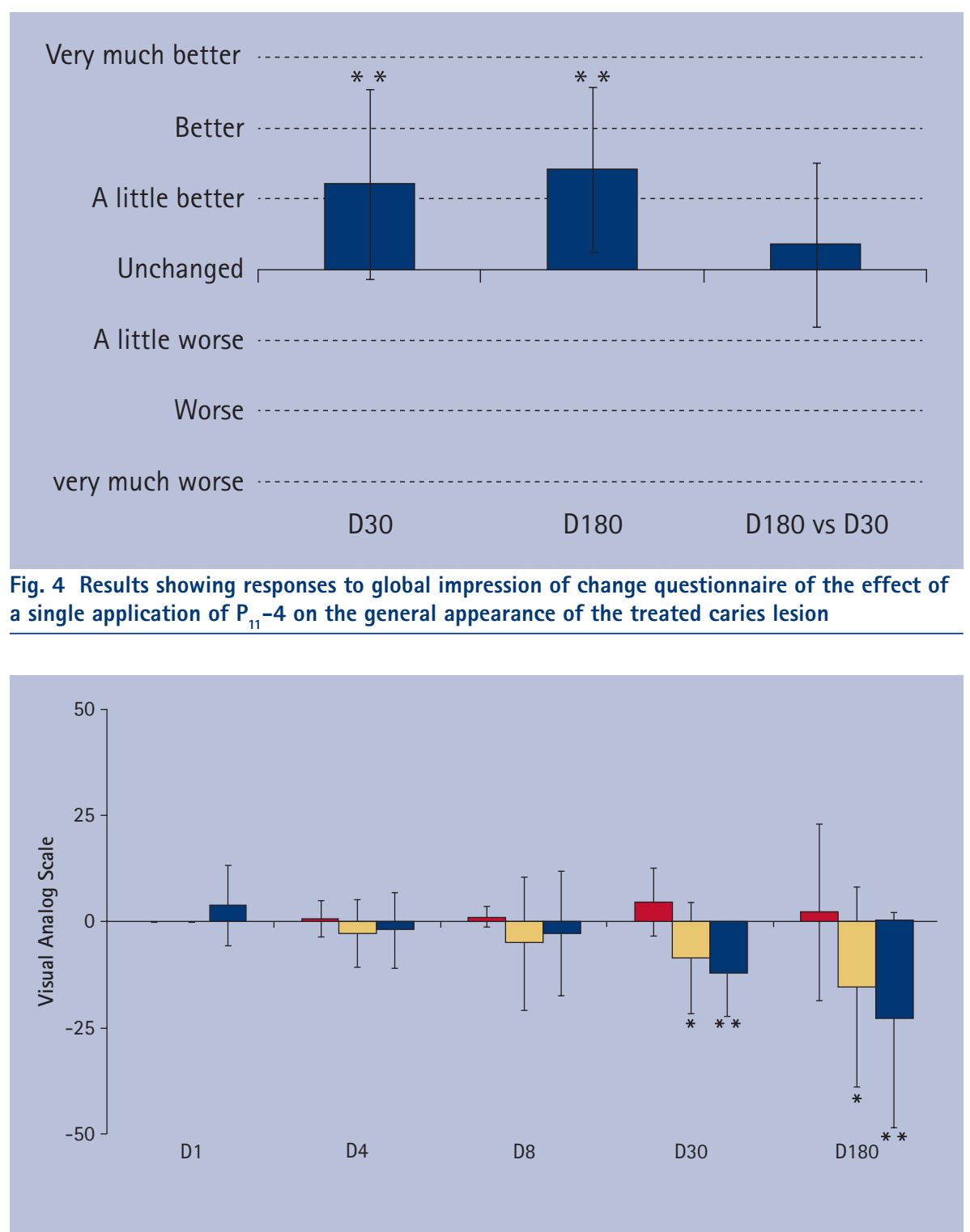

Fig 5. Effect of a single application of $\mathrm{P}_{11}-4$ on colour, size and progression of the treated caries lesion compared to baseline (DO) as judged by blinded assessors using a visual analogue scale from -100 to +100 . Histograms show change in lesion colour (red); change in lesion size (yellow) and change in lesion progression (blue). Lesion colour: positive values indicate an improvement in lesion colour (ie less white). Lesion size: positive values indicate a lesion that is increasing in size; negative values a decrease in lesion size. Lesion progression: positive values indicate a lesion that is judged to be progressing, negative values indicate a lesion that is judged to be remineralising. Significance when compared with baseline indicated by * $(p \leq 0.05) ;{ }^{* *}(p \leq 0.001)$

be not related to the study. None of the adverse events were classified as serious.

The effect of treating the lesions using $\mathrm{P}_{11}-4$ was initially assessed based upon their clinical appearance using clinical photographs (Fig. 3). Statistical analysis of the scoring by the two assessors showed no significant differences in their assessments across all of the measured outcomes.

The results of the clinicians' global impression of change questionnaire are shown in Figure 4. Compared with baseline, there was a significant improvement in the assessment scores 30 days after a single application of $\mathrm{P}_{11}-4(\mathrm{p}<0.001)$.
This improvement was maintained even after 180 days post-treatment; results compared with baseline showed a highly significant improvement in the global impression of change scores ( $p<0.0001$ ). No statistically significant differences in the global impression of change scores were seen when D180 was compared with D30, suggesting that the major treatment effect for $\mathrm{P}_{11}-4$ had occurred by D30 post-treatment.

A VAS was used to assess specific lesion parameters including colour, size and progression based upon the appearance of the lesion in the clinical photographs.
Figure 5 summarises the VAS data for each of the follow up visits compared with. baseline. No significant differences were seen in any of the three parameters in the first follow up visit (D4). By D8, there was a significant difference observed in lesion colour ( $\mathrm{p}<0.05$ ) but this was not significant at any of the later time points, mainly due to the very large variation in the scoring. For lesion size and progression, there was an increasing trend towards improvement over the first eight days after treatment but this was not statistically significant until D30, where lesion size was judged to have significantly decreased ( $p<0.05$ ) and lesion progression was judged to have moved from arrested/progressing to remineralising $(\mathrm{p}<0.01)$. This improvement in lesion size and progression was maintained at D180 (almost six months after the single application of $\mathrm{P}_{11}-4$ ).

Finally, in agreement with the clinical assessment, morphometic analysis also showed a significant decrease in lesion size compared with baseline at D30 (31 $\pm 24 \%$, $\mathrm{n}=11 ; \mathrm{p}=0.002)$ and D180 (40 $\pm 27 \%$, $\mathrm{n}=11 ; \mathrm{p}=0.001$ ).

\section{DISCUSSION}

This small study represents a first-in-man clinical safety trial of a regenerative caries therapeutic that has been rationally designed to recapitulate the processes occurring during enamel biomineralisation, where a self-assembled organic matrix controls the deposition and growth of hydroxyapatite crystals. ${ }^{13}$ As a potential treatment for early caries lesions, $\mathrm{P}_{11}-4$ is safe, non-invasive and acceptable to patients (as evidenced by the unanimous and significantly positive response on the patients' global impression of change questionnaire [ $=0.0003]$ ). However, it is important to note that this is not an efficacy trial and as such we cannot compare the data obtained here with a 'do nothing' control or any other intervention, such as the application of topical fluoride.

We have previously shown that $\mathrm{P}_{11}-4$ is able to nucleate hydroxyapatite de novo and is able to promote repair of carieslike lesions in vitro. ${ }^{1}$ The treatment differs from other 'filling without drilling' infiltrative strategies in that $\mathrm{P}_{11}-4$ is a bioactive peptide synthesised from natural amino acids that is triggered to assemble into a three dimensional fibrillar scaffold 
by environmental conditions of $\mathrm{pH}$ and salt concentration. Assembly takes place within the lesion, and the scaffold can then act as nucleator for hydroxyapatite, effecting tissue regeneration from within. Infiltration of lesion pores using low viscosity resins such as sealants has been demonstrated to halt caries progression in vitro, ${ }^{19,20}$ in situ ${ }^{21}$ and in vivo, ${ }^{22}$ presumably by providing a barrier to diffusion within the lesion. The use of a biomimetic peptide such as $\mathrm{P}_{11}-4$ has the additional advantage of effecting 'natural' repair by regenerating the mineral itself.

We used a global impression of change questionnaire coupled with assessment of clinical photographs using VAS and morphometric analysis of lesion size to determine any clinical benefit following treatment of the lesions with $\mathrm{P}_{11}-4$. Visual analogue scales are commonly used in dental research, for example in judging severity of fluorosis. ${ }^{23}$ We chose not to use radiographs as an outcome measure as the changes we were seeking to identify are relatively small, given that these are early lesions which do not themselves necessarily show on radiographs. We chose not to use caries detection devices as those currently available depend on surrogate indicators of lesion progression/detection (eg QLF depends on the enamel structure; Diagnodent depends on presence of porphyrins; CarieScan depends on conductivity or water) though it would be interesting to utilise these diagnostics in future efficacy trials. The results of the study indicate that the clinical use of $\mathrm{P}_{11}-4$ is safe and, based upon clinical judgement, Class V lesions treated with a single application of $\mathrm{P}_{11}-4$ show significant improvement in their clinical appearance by D30. This improvement in size and progression was maintained even after 180 days post-treatment and was further supported by the morphometric analysis. We do not know the kinetics of peptide-induced remineralisation but the data suggests a safe and positive trend beginning at the earliest days post-treatment. It is also possible that the potential for lesion repair might be influenced by the initial state of the lesion itself. We have no historical data relating to those lesions that were treated here and the small sample size means that we cannot make any evaluation as to whether initial lesion activity is related to treatment outcome. This would need to be addressed as part of a more comprehensive efficacy trial.

Despite the fact that this study was a small, non-controlled safety clinical trial, all of the outcome measures used showed beneficial results in respect of enamel regeneration. The results are therefore promising given that we have not yet investigated the optimum clinical delivery for $\mathrm{P}_{11}-4$ or the formulation of $\mathrm{P}_{11}-4$ itself. It may be that we can improve efficacy by pre-treating lesions to remove extraneous material, such as proteins that may be present within. ${ }^{24}$ In addition, we have yet to determine whether repeated application of $\mathrm{P}_{11}-4$ would provide further enamel benefit for those lesions where repair was incomplete. Given that $\mathrm{P}_{11}-4$ is a welltolerated treatment, repeated application is an obvious way forward. Finally, we are currently designing and bench testing 'next generation' peptides to accelerate the repair process, making 'filling without drilling' a reality.

Supported by credentis ag, Switzerland and in part by WELMEC, a Centre of Excellence in Medical Engineering funded by the Wellcome Trust and EPSRC; WT 088,908/Z/09/Z. JK is supported by the NIHR Leeds Musculoskeletal Biomedical Research Unit.

Conflict of interest declaration: J. Kirkham and A. Aggeli are named inventors on the underlying patent on self-assembling peptides. The University of Leeds holds an equity stake in credentis.

1. Kirkham J, Firth A, Vernals D et al. Self-assembling peptide scaffolds promote enamel remineralization. J Dent Res 2007; 86: 426-430.

2. Aggeli A, Bell M, Boden N et al. Responsive gels formed by the spontaneous self-assembly of peptides into polymeric beta-sheet tapes. Nature 1997 386: 259-262.

3. Aggeli $A$, Bell $M$, Boden $N$ et al. Engineering of peptide beta-sheet nanotapes. J Mater Chem 1997; 7: 1135-1145.

4. Aggeli $A$, Bell $M$, Carrick $L M$ et al. $\mathrm{pH}$ as a trigger of peptide beta-sheet self-assembly and reversible switching between nematic and isotropic phases. J Am Chem Soc 2003; 125: 9619-9628.
5. Aggeli A, Fytas G, Vlassopoulos D et al. Structure and dynamics of self-assembling beta-sheet peptide tapes by dynamic light scattering. Biomacromolecules 2001; 2: 378-388.

6. Aggeli A, Nyrkova I A, Bell M et al. Hierarchical selfassembly of chiral rod-like molecules as a model for peptide beta -sheet tapes, ribbons, fibrils, and fibres. Proc Natl Acad Sci U S A 2001; 98: 11857-11862.

7. Firth A, Aggeli A, Burke J L, Yang X, Kirkham J. Biomimetic self-assembling peptides as injectable scaffolds for hard tissue engineering. Nanomedicine (Lond) 2006; 1: 189-199.

8. Kitasako Y, Hiraishi N, Nakajima M et al. In vitro surface analysis of active and arrested dentinal caries using a $\mathrm{pH}$-imaging microscope. Oper Dent 2002; 27: 354-359.

9. Burke J L. In situ engineering of skeletal tissues using self-assembled biomimetic scaffolds [PhD]. Leeds: The University of Leeds, 2011.

10. Felton S. Self assembling -sheet peptide networks as smart scaffolds for tissue engineering [PhD Thesis]. Leeds: University of Leeds, 2005.

11. Kirkham J, Brookes S J, Shore R C et al. Physicochemical properties of crystal surfaces in matrix- mineral interactions during mammalian biomineralisation. Curr Opin Colloid In 2002; 7: 124-132.

12. Wen H B, Moradian-Oldak J, Leung W, Bringas P Jr., Fincham A G. Microstructures of an amelogenin gel matrix. J Struct Biol 1999; 126: 42-51.

13. Simmer J P. Fincham A G. Molecular mechanisms of dental enamel formation. Crit Rev Oral Biol M 1995; 6: 84-108.

14. Kim J W, Seymen F, Lin B P et al. ENAM mutations in autosomal-dominant amelogenesis imperfecta. J Dent Res 2005; 84: 278-282

15. Wright J T, Hart P S, Aldred M J et al. Relationship of phenotype and genotype in X-linked amelogenesis imperfecta. Connect Tissue Res 2003; 44: 72-78.

16. Robinson C, Kirkham J, Brookes S J, Shore R C. Chemistry of mature enamel. In Robinson $\mathrm{C}$, Kirkham J, Shore R C (eds) Dental enamel formation to destruction. pp 167-191. Boca Raton: CRC Press, 1995.

17. Robinson C, Shore R C, Brookes S J et al. The chemistry of enamel caries. Crit Rev Oral Biol M 2000; 11 481-495.

18. Johnson A R. The early carious lesion of enamel. J Oral Pathol 1975; 4: 128-157.

19. Fan $P L$, Seluk $L W, O$ 'Brien $W J$ J. Penetrativity of sealants: I. J Dent Res 1975; 54: 262-264.

20. Robinson C, Brookes S J, Kirkham J, Wood S R, Shore R C. In vitro studies of the penetration of adhesive resins into artificial caries-like lesions. Caries Res 2001; 35: 136-141.

21. Paris S, Meyer-Lueckel H. Inhibition of caries progression by resin infiltration in situ. Caries Res 2010; 44: 47-54.

22. Paris S, Hopfenmuller W, Meyer-Lueckel H. Resin infiltration of caries lesions: an efficacy randomized trial. J Dent Res 2010; 89: 823-826.

23. Vieira A P, Lawrence $H$ P, Limeback $H$, Sampaio F C, Grynpas M. A visual analogue scale for measuring dental fluorosis severity. J Am Dent Assoc 2005; 136: 895-901.

24. Shore R C, Kirkham J, Brookes S J, Wood S R, Robinson $C$. Distribution of exogenous proteins in caries lesions in relation to the pattern of demineralisation. Caries Res 2000; 34: 188-193.

25. Kay MI, Young R A, Posner A S. Crystal structure of hydroxyapatite. Nature 1964; 204: 1050-1052.

26. Elliott J C. Structure and chemistry of the apatites and other calcium orthophosphates. Amsterdam: Elsevier, 1994. 